\title{
The API Spatial and Temporal Change Analysis of Key Environmental Protection Cities in China Based on $\mathrm{EOF}$
}

\author{
Liu Bai-qiao \\ Urban and Environmental Science College \\ Tianjin Normal University \\ Tianjin, China \\ liu_baiqiao@263.net
}

\author{
Liao Shan-yun \\ jie_4428@163.com
}

\begin{abstract}
In this paper, monthly averaged API index data from August 2006 to September 2009 of the $86 \mathrm{key}$ environmental protection cities in China were broken down into several fields and their time functions by the Empirical Orthogonal Function (EOF) method, and the temporal and spatial distribution characteristics of API were analyzed. It was shown that the regional differences of API were significant among major Chinese environmental protection cities, and the 86 key cities may be divided into 8 areas. The analysis by the EOF could effectually reflect the spatial-temporal distinctly differences of API distributions, which may provide new methods and ideas to study regional situations of air pollution.
\end{abstract}

Keywords-Air Pollution Index(API); Empirical Orthogonal Function(EOF); Spatial and temporal distribution

\section{INTRODUCTION}

Since the 20th century, 90 years, along with the rapid development of Chinese national economy, the regional characteristics of urban air quality was increasingly evident led by urbanization and city circle expansion, and receive extensive attention ${ }^{[1]}$. The main problem of atmospheric environment (especially in urban ambient air quality) was particulate pollution. Air Pollution Index (API) distribution was subjected to weather conditions, topography, people's status of activities and many other factors which have complex spatial and temporal distribution characteristics. The study of API temporal and spatial distribution characteristics is positive significance to urban air quality analysis and prediction.

At present, there were little researches on countrywide or regional air pollution index. Most research are largely on time series, and some also discuss the role of meteorological factors: Tian Liang et al.(2005) ${ }^{[2]}$ researched Chinese major cities air pollution temporal and spatial distribution with journal data of urban air quality from 2001 to 2004, which issued by State Environmental Protection Administration. The regional pollution studies had been done by a lot of authors: Ren Zhenhai et al.(2004) $)^{[3]}$ analyzed Chinese atmospheric environment monitoring data from 1998 to 2002, making out that air quality distribution had shown large regional characteristics. More research works were done on one city's air pollutants or air pollution index (sub-index) changes: $\mathrm{Si}$ Bingyao et al. $(2005)^{[4]}$, using air pollutants motoring data (TSP, $\mathrm{SO}_{2}, \mathrm{NO}_{\mathrm{x}}$ ), 1990-2002, in Hohhot, found that urban

IEEE Eng. in Medicine and Biology Society, USA

Sichuan University, China

Wuhan University, China

Scientific Research Publishing, USA

Centre for Advanced Water Technology, Singapore Public Utilities Board, Singapore pollution was mainly caused by coal-burning, and the pollution is more serious in winter and spring than in summer and autumn, in the heating period than the non-heating period. There were little studies on the temporal and spatial distribution of air pollution, especially on large-scale of the regional distribution characteristics. The Empirical Orthogonal Function (EOF) method is an important way to analyze the data dimensionality of space-time. It was wisely applied in the meteorological field, mainly used to study the temporal and spatial distribution laws of meteorological element fields, such as Zhu Min (2004) ${ }^{[5]}$ implied EOF to decompose NCEP data of the South China Sea summer monsoon in 1983 1992, and built a main modal characteristics of space-time transformation. Liu Yulu (2007) ${ }^{[6]}$ used EOF to decompose the rainfall data during rainy season sequence of Huangshan in recent 45 years, and got its temporal and spatial distribution characteristic. However, there are not many researches on application of atmospheric environment.

In this paper, the API data of nation's key environmental protection were analyzed by EOF method to discuss the geographical distribution of regional air pollution characteristics and inter-annual variation. It was expected that the deep excavation of the data can reflect the regional characteristics of Chinese air pollution, and reveal their change laws in long term.

\section{DATA AND METHODS}

\section{A. Data and sources}

Since June 2000, National Environmental Monitoring Center organized 42 key environmental protection cities to do daily reporting and forecasting urban air quality work ${ }^{[7]}$, according to relevant requirements from the State Environmental Protection Administration. June 2001, the key environmental protection cities in China increased to 47, June 2004, increased to 84 , and January 2006, increased to 86 .

In this paper, the analyzed API data were form $86 \mathrm{key}$ environmental protection cities in 2006.8-2009.9, which released by the National Environmental Monitoring Center ${ }^{1}$.

\footnotetext{
${ }^{1}$ http://datacenter.mep.gov.cn/TestRunQian/air_dairy.jsp
} 


\section{B. Research Methods}

EOF is an important method of multivariate statistical analysis, which based on a large number of measured data in the analysis of many elements on the market. The element field was decomposed to series typical feature vectors presenting as time-dependent functions and space functions ${ }^{[8]}$, which objectively and quantitatively reflect the element markets. The basic principle is decomposing the element market $\left(\mathrm{F}_{\mathrm{ij}}\right)$ into orthogonal functions of time $\left(\mathrm{T}_{\mathrm{ip}}\right)$ and orthogonal spatial functions $\left(\mathrm{X}_{\mathrm{pj}}\right)^{[9]}$. That is:

$$
F_{\mathrm{ij}}=\sum\left(T_{\mathrm{ip}} \times X_{\mathrm{pj}}\right)
$$

Where $F_{i j}$ as the element market, $\mathrm{i}=1,2 \ldots, \mathrm{m}$, is the time serial number, $\mathrm{j}=1,2, \ldots \mathrm{n}$, is the site number; $X_{\mathrm{pj}}$ is space function, $\mathrm{h}=1,2, \ldots \mathrm{P}$, is the number of decomposition field; $\mathrm{T}_{\mathrm{ip}}$ is a function of time.

Space function $\mathrm{X}_{\mathrm{pj}}$ usually was regarded as a typical field. It was entirely changes with spatial, but not changed with time. The time function $T_{i p}$ contained the only time-varying function, regarded as a typical market weight factor.

Ordinary, EOF convergence quickly, and the sum of prior few typical feature vectors could able to represent the actual field in general. The value of time function reflected importance of typical market in different times.

Format (1) could be written in matrix form, namely:

$$
\left|\begin{array}{cccc}
F_{11} & F_{12} & \cdots & F_{1 \mathrm{n}} \\
F_{21} & F_{22} & \cdots & F_{2 \mathrm{n}} \\
\cdots & \cdots & \cdots & \cdots \\
F_{m 1} & F_{m 2} & \cdots & F_{m n}
\end{array}\right|=\left|\begin{array}{cccc}
T_{11} & T_{12} & \cdots & T_{1 \mathrm{n}} \\
T_{21} & T_{22} & \cdots & T_{2 \mathrm{n}} \\
\cdots & \cdots & \cdots & \cdots \\
T_{m 1} & T_{m 2} & \cdots & T_{m n}
\end{array}\right| \begin{array}{cccc}
x_{11} & x_{12} & \cdots & x_{1 \mathrm{n}} \\
x_{21} & x_{22} & \cdots & x_{2 \mathrm{n}} \\
\cdots & \cdots & \cdots & \cdots \\
x_{n 1} & x_{n 2} & \cdots & x_{n n}
\end{array} \mid
$$

The variability distribution structure of a regional API field was characterized by typical feature vectors. Their spatial distribution forms, characterized by space functions, represented the main body's distribution of the variable field, while correspondence time functions represented time-varying characteristics of regional distribution forms.

The positive coefficient of time function indicated that the distribution form was the major change trend, and the negative coefficient indicated that the distribution form was the opposite change trend. The coefficient value is higher; the distribution form of corresponding time is more significant ${ }^{[10]}$.

\section{RESULTS}

\section{A. Spatial Distribution of API Index}

The matrix $\mathrm{X}$ was decomposed by EOF, which is made by API data from 86 key environmental protection cities, in 2006.8-2009.9. Characteristic values of the prior four typical feature vectors were shown in Table 1.The cumulated contribution rate of first and second typical feature vectors were $97.8 \%$ and $98.5 \%$, indicating that API distribution of national key environmental protection cities mainly changed with the trends of prior two typical feature vectors in the spatial distribution. Therefore, taking on the prior two typical feature vectors could achieve a very good fit to original market.
Tab.1 Characteristic values of the prior four typical feature vectors

\begin{tabular}{|c|c|c|}
\hline \multirow{2}{*}{$\begin{array}{c}\text { Serial } \\
\text { Number }\end{array}$} & \multicolumn{2}{|c|}{ Eigenvalue and Cumulative Contribution } \\
\cline { 2 - 3 } & Eigenvalue & Cumulative Contribution \\
\hline 1 & 16452703.09 & 0.978 \\
\hline 2 & 122820.78 & 0.985 \\
\hline 3 & 44934.72 & 0.988 \\
\hline 4 & 29625.68 & 0.989 \\
\hline
\end{tabular}

It can be seen from Tab. 1 that the first typical feature vector variance contribution was $97.8 \%$ of the total variance, which is much larger than variance contributions of other typical feature vectors. The spatial distribution form of the first typical feature vector can be seen in Fig.1, which had both positive and negative values. It was shown that there were obvious spatial differences of API in China. The main value of spatial distribution form is positive, and the positive value concentrated in the Henan-Shanxi region, the middle area of Yangtze River region, Northeast China region and the coastal areas of Fujian region. Negative area mainly concentrated in the Beijing-Tianjin-Tangshan area, the Shandong peninsula region, Tibet and southern China region.

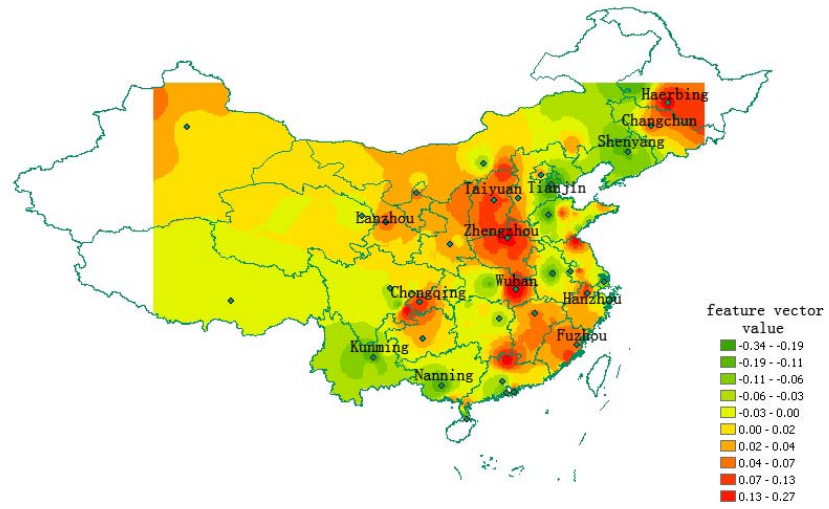

Fig.1 Spatial distribution of first feature vectors

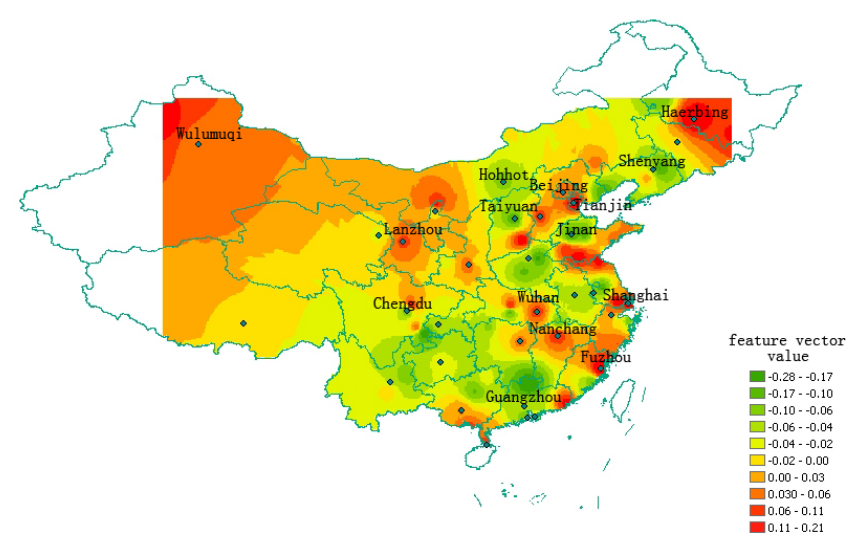

Fig.2 Spatial distribution of second feature vectors

The second typical feature vector variance contributed rate was $0.8 \%$ of the total variance, which may reflect minor distribution characteristics of API changes. The spatial distribution form of the first typical feature vector can be seen in Fig.2, which also had both positive and negative values. It was shown that the positive area mainly concentrated in Xinjiang region, the Northeast China region, the Yellow River region, the Beijing-Tianjin-Tangshan, the middle area of Yangtze River region, the Delta of Yangtze River region, the 
Shandong peninsula region and the coastal areas of Fujian region. The negative value area focused on Southwest China region, south China region, and Anhui-Henan region.

\section{B. Time-varying Characteristics of API Index}

Time coefficients, decomposed from EOF, represented time-varying characteristics of distribution forms. Fig.3 showed time coefficient curve for the first typical feature vector of API. From Fig.3 we can see that the value of first time coefficient was positive all the time, indicating that the API distributions of key environmental protection cities had a relatively stable pattern as shown in Fig. 1 in long time. API had visible annual variation characteristics which reflected the influences mainly from climate alternating. In the first half of a year, 2007.1 to 2007.7, 2008.2-2007.7 and 2009.1-2009.6 API were gradually falling down, while in the second half of year, 2006.8-2006.12, 2007.8-2007.12 and 2008.8- 2008.12 API were gradually rising. Annual maximums and minimums of time coefficient decreased from 2006-2009. In 2009.6 API reached minimum.

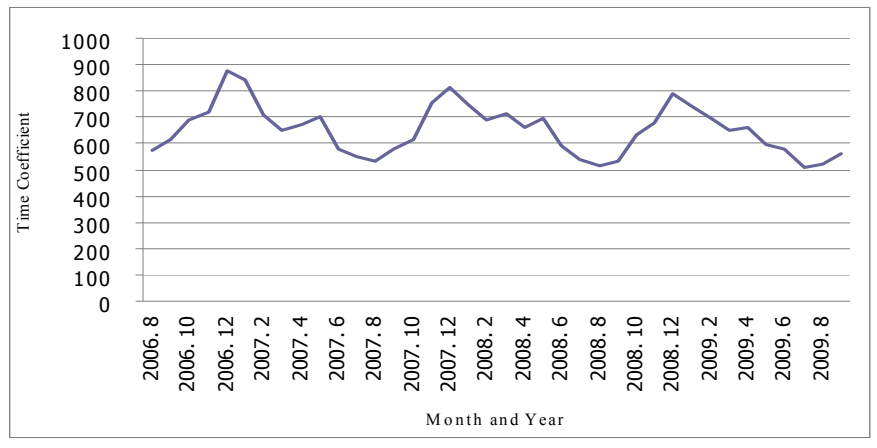

Fig.3 Time coefficient variation of the first typical feature vector

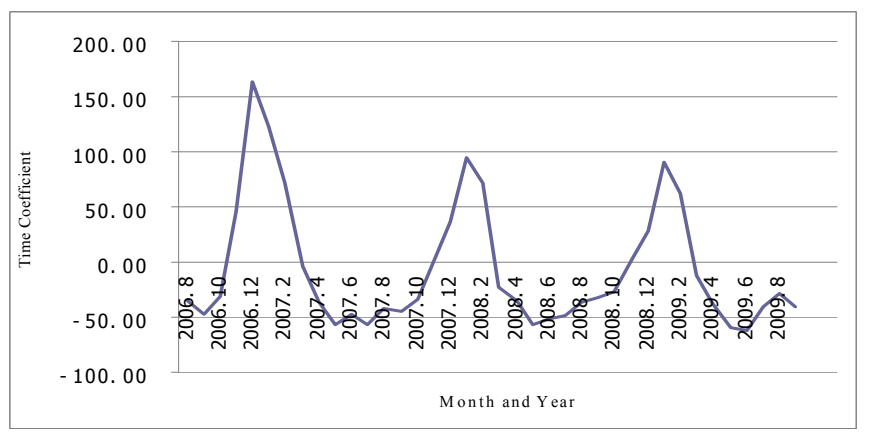

Fig.4 Time coefficient variation of the second typical feature vector

Fig.4 showed time coefficient curve for the second typical feature vector of API. It was shown that API was positive in winter, 2006.11-2007.3, 2007.11-2008.3 and 2008.11-2009.3, and was negative in other time. It may indicate influence from climate and urban heat supplying.

\section{DISCUSSION}

EOF method was used to decompose 3-year monthly averaged API of 86 key environmental protection cities nationwide, in 2006.8-2009.9. Based on the result of API space functions and time functions, the characteristics of spatial distribution and time coefficient of national API were analyzed.

According to the analysis results, it can be seen that there was clear temporal regulations and spatial distribution structures of urban air pollution in China. The main characterized regions were eight large areas: southwest China, the middle area of Yangtze River region, the Delta of Yangtze River region, Beijing-Tianjin-Tang, the Shandong peninsula region, Tibet, and South China and Northeast China; there was a significant time-varying regulation indicating that API index was higher in winter, lower in summer and autumn.

This paper just analyzed the past three year's data of the API by EOF. To obtain more universal spatial and temporal distribution regulations of air pollution in China, we need to extend time series of API data.

\section{CONCLUSIONS}

1) The prior two typical feature vectors from EOF could achieve a very good fit to API distribution of national key environmental protection cities ;

2) The country could be divided into eight characterized regions of urban air pollution according to the prior two typical feature vectors;

3) API had visible annual variation characteristics which reflected the influences mainly from climate alternating and urban heat supplying;

4) Annual maximums and minimums of time coefficient decreased from 2006-2009.

\section{REFERENCES}

[1] Xiang Min, Han Yongxiang, Deng Zuqin. Spatial-temporal Distribution Characteristic of Chinese Cities Air Pollution in 2007[J].Environmental Monitoring Management and Technogy, 2009, pp.33-36.

[2] Tian Liang, Lu Ranying, XingWenting. Studies on City Ambient Air Quality in China During $2001 \sim 2004[\mathrm{~J}]$.Arid Land Resources and Environment, 2005, pp.101-105.

[3] Ren Zhenhai, Wan Bentai, Su Fuqing. Several Characteristic s of Atmospheric Environmental Quality in China at Present[J]. Environmental Sciences, 2004, pp.3-8.

[4] Si Yaobing, Gong Chunning, Zheng Youfei. Correlation Between Air Pollution Density and Climate in Hohhot [J]. Meteorological Science and Technology, 2005, pp.173-177.

[5] Zhu Min, Zhang Min.EOF Expansion of Summer Monsoon Onset over the South China Sea[J].Meteorology,2004, pp.261-268.

[6] Liu Yulu, Xu Bolin, Xu Jianyong. Spatial-Temporal Characteristics of Precipitation in Flood Season in Huangshan Mountains [J]. Meteorology, 2007, pp.47-51.

[7] Tong Yanchao. Air Quality Forecast and Development in Major Cities of China[J]. China Environmental Monitoring, 2006, pp.69-71.

[8] Zhu Liangsheng. An Analysis of Sound Velocity in Waters of Zhujiang River Estuary with the Empirical Orthogonal Function (EOF)[J].Taiwan Strait,2006, pp.181-187.

[9] Cui Duxin. Application of Empirical Orthogonal Function Resolution to Analysis of Crustal Vertical Deformation Field [J]. Earthquake, 2000, pp.82-86.

[10] DuanYusen, Wen Haiping, FuQingyan. Regional Spatio-temporal mode differences of Air Pollution Index of Key Environmental Protection Cities in China[J].Journal of Environmental Science,2008, pp.384-391. 